\title{
Stopping power of cluster ions in a free-electron gas from partial-wave analysis
}

\author{
F. Matias, R. C. Fadanelli, and P. L. Grande \\ Ion Implantation Laboratory, Institute of Physics, Federal University of Rio Grande do Sul, Av. Bento Gonçalves, 9500, CP 15051, \\ CEP 91501-970, Porto Alegre, RS, Brazil \\ N. R. Arista \\ División Colisiones Atómicas, Instituto Balseiro and Centro Atómico Bariloche, Comisión Nacional de Energía Atómica, \\ 8400 San Carlos de Bariloche, Argentina
}

\author{
N. E. Koval \\ Donostia International Physics Center (DIPC), Paseo Manuel de Lardizabal 4, E-20018 Donostia-San Sebastián, Spain \\ and Centro de Física de Materiales CFM/MPC (CSIC-UPV/EHU), Paseo Manuel de Lardizabal 5, E-20018 Donostia-San Sebastián, Spain \\ G. Schiwietz \\ Abteilung Betrieb Beschleuniger BESSY II (NP-ABS), Helmholtz-Zentrum Berlin für Materialien und Energie GmbH, Hahn-Meitner-Platz 1, \\ D-14109 Berlin, Germany
}

(Received 3 August 2018; published 28 December 2018)

\begin{abstract}
A nonlinear model for the stopping power of cluster ions based on partial-wave analysis is developed through the generalization of the induced density approach (IDA) model for the interaction of homo- and heteronuclear molecular ions with a free-electron gas (IDAMol). We apply IDAMol to the energy loss of $\mathrm{H}_{2}{ }^{+}$dimers in $\mathrm{SiO}_{2}$ and $\mathrm{Al}_{2} \mathrm{O}_{3}$, where we find that the results are consistent with established linear (dielectric) models at higher speeds, as expected for small perturbations (small values of the projectile charge or high velocities). Specifically at low projectile energies, however, it is important that IDAMol goes beyond perturbation theory. This feature appears to be central for a good description of negative and positive vicinage effects, a measure of the deviation from the independent-atom model. The focus of this work, however, is the investigation of enhanced nonlinear effects. Here we present experimental results for a heteronuclear cluster ion namely $\mathrm{HeH}^{+}$on $\mathrm{Al}_{2} \mathrm{O}_{3}$, in the energy range of few tens of $\mathrm{keV} / \mathrm{u}$ using the medium energy ion scattering technique. The IDAMol results are corroborated by the experimental data and time-dependent density-functional calculations for this case. Strong nonlinearities are observed for the energy loss of the fragment $\mathrm{H}^{+}$due to the higher charge of its He companion.
\end{abstract}

DOI: 10.1103/PhysRevA.98.062716

\section{INTRODUCTION}

The energy loss of cluster fragments after breakup in matter is different from the sum of energy losses of each fragment as long as they are traveling together [1-8]. This phenomenon is known as the vicinage effect and has been investigated for many years since it could be important to generate intensive x-ray sources, to drive nuclear fusion [9], and to perform ion-beam analysis for ultrathin films [10]. For $\mathrm{keV}$ light dimers most investigations have been done using the framework of dielectric function formalism. Notable exceptions are recent time-dependent density functional theory (TDDFT) calculations [11-13] and the oscillator model for hydrogen diclusters [14].

For energies larger than tens of $\mathrm{keV}$ and for light cluster ions, the energy loss is dominated by electronic ionization and excitation of the medium [15]. The vicinage effect can be then understood as an interference effect on the ionization and excitation of the medium after a sequence of atomic collisions or due to superposition of wake potentials generated by each fragment [5]. The ratio $R$ between the cluster energy loss and the sum of energy losses of each fragment in a target is usually larger than 1 for larger beam energies (positive interference) and lower than 1 (negative interference) for smaller beam energies. For instance, as reported for the $\mathrm{H}_{2}{ }^{+}$cluster in $\mathrm{C}$ [16] and $\mathrm{Al}$ [17], and $\mathrm{H}_{2}{ }^{+}$and $\mathrm{H}_{3}{ }^{+}$clusters in $\mathrm{SiO}_{2}$ [15], the transition between negative and positive interference can be abrupt around the threshold energy for plasmon excitation, indicating the importance of collective effects of the target electrons for the stopping power. Calculations based on the dielectric function of an ensemble of harmonic oscillators [14], which can produce negative and positive interference, could not, however, explain this transition.

Recently, an approach has been developed to evaluate the electronic stopping power and the transport cross section in electron-ion collisions, namely the induced density approach (IDA) and applied to $\mathrm{H}^{+}$ions in a free-electron gas (FEG) $[18,19]$. The stopping power is calculated by the retarding force caused by the asymmetric induced charge density on the projectile. For this sake a central Yukawa potential $V(r)$ generates the noncentral induced charge density $n_{\text {ind }}(\vec{r})$ from the partial-wave expansion of the stationary wave function for the electron-ion collision in the rest frame of the ion. In this 
procedure, $n_{\text {ind }}(\vec{r})$ is used to evaluate either the noncentral induced potential $V_{\text {ind }}(\vec{r})$ or the induced force $\vec{F}_{\text {ind }}(\vec{r})$ on the ion position, leading, therefore, to the electronic stopping power. The IDA results show a better convergence to the Bethe formula for high energies than the usual transport crosssection (TCS) procedure [20-22].

In this work, we generalize the IDA for cluster ions and calculate the vicinage effect on the stopping power for $\mathrm{H}_{2}{ }^{+}$ ions in different oxides from the literature. We also provide measurements of the vicinage effects for heteronuclear $\mathrm{HeH}^{+}$ cluster ions. If not indicated otherwise atomic units $(\hbar=$ $m_{e}=e=1$ ) are used throughout this paper.

\section{THEORETICAL PROCEDURE}

\section{A. Point charged stopping power}

For point charged particles the electronic stopping power can be calculated from the induced force $\vec{F}_{\text {ind }}$ at the projectile $(\vec{r}=0)$ as

$$
\begin{aligned}
\frac{d E}{d z} & =-\frac{1}{v} \vec{F}_{\text {ind }} \cdot \vec{v} \\
& =-Z\left[\int \frac{\partial}{\partial z}\left(\frac{n_{\text {ind }}\left(\vec{r}^{\prime}\right)}{\left|\vec{r}-\vec{r}^{\prime}\right|}\right) d^{3} r^{\prime}\right]_{\vec{r}=0},
\end{aligned}
$$

where $Z$ is the nuclear charge, $\vec{v}$ is the particle velocity and $n_{\text {ind }}(\vec{r})$ is the induced electron density, which reads for an freeelectron gas (FEG) system in the reference frame where the projectile is at rest [23]:

$$
n_{\text {ind }}(\vec{r})=\frac{2}{(2 \pi)^{3}} \int_{\mathrm{DFS}}\left(\left|\psi_{\vec{k}}\right|^{2}-1\right) d^{3} k,
$$

where the $\vec{k}$ integration is performed over the displaced Fermi sphere (DFS) [23-25]. For a central electron-ion potential $V(r)$ the induced density $n_{\text {ind }}(\vec{r})$ can be calculated from the partial-wave expansion of the stationary wave function [26],

$$
\psi_{\vec{k}}(\vec{r})=4 \pi \sum_{\ell, m} i^{\ell} e^{i \delta_{\ell}} \mathcal{R}_{k, \ell}(r) Y_{\ell, m}(\hat{r}) Y_{\ell, m}^{*}(\hat{k}),
$$

with $\vec{k}$ being the the incident electron momentum, $\mathcal{R}_{k, \ell}(r)$ the radial continuum wave function with angular-momentum quantum-number $\ell$, and $Y_{\ell, m}$ the spherical harmonics of $\hat{r}$ and $\hat{k}$, directions of $\vec{r}$ and $\vec{k}$, respectively, and $m$ the azimuthal quantum number $(|m| \leqslant \ell)$. The phase shifts $\delta_{\ell}$, obtained from the asymptotic limit $(r \rightarrow \infty)$ of the radial wave function as

$$
\mathcal{R}_{k, \ell}(r) \rightarrow \frac{1}{r} \sin \left(k r-\ell \frac{\pi}{2}+\delta_{\ell}\right),
$$

depend on the scattering energy or, more accurately, on the asymptotic electron momentum $k$ and on the influence of the potential $V(r)$ on each partial wave.

The stopping power Eq. (1) can be written in terms of the phase shifts from the scattering potential $V(r)$ as demonstrated recently in Ref. [18] and named the induced-density

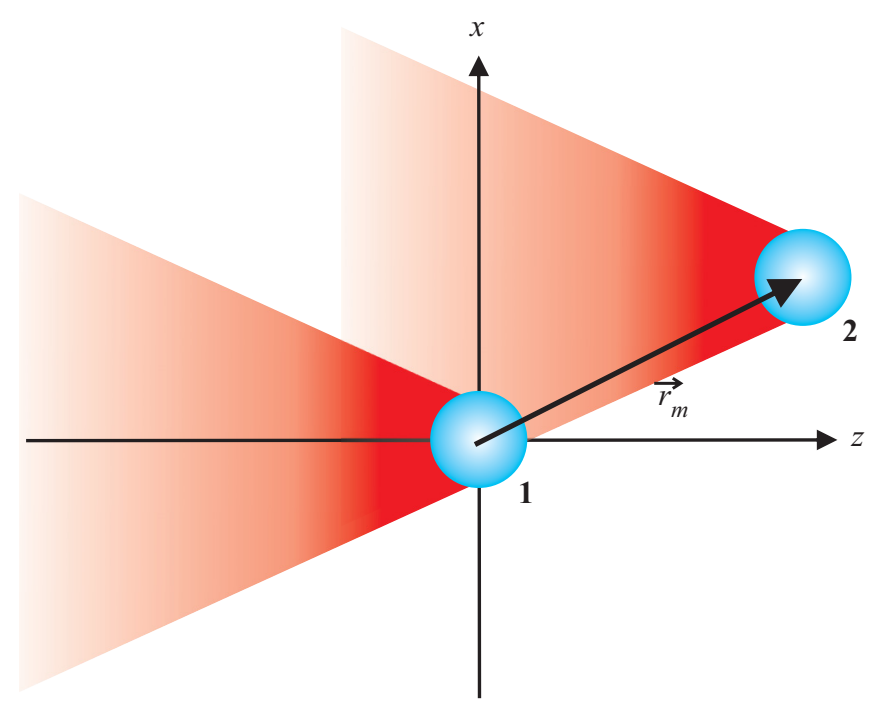

FIG. 1. Sketch of the induced potential from two charged particles.

approach (IDA),

$$
\begin{aligned}
\frac{d E}{d z}= & \frac{Z}{8 \pi v^{2}} \int_{\left|v_{F}-v\right|}^{v_{F}+v} \frac{d k}{k} \\
& \times\left(2 k^{2}\left(v_{F}^{2}+v^{2}\right)-k^{4}-\left(v_{F}^{2}-v^{2}\right)^{2}\right) \\
& \times \sum_{\ell=0}^{\infty} \sin \left(2\left(\delta_{\ell}(k)-\delta_{\ell+1}(k)\right)\right)
\end{aligned}
$$

which is notably different from the transport cross-section (TCS) approach, which is based on the momentum transfer cross section [20-22]. Both formulas are nonperturbative and able to describe nonlinear effects on the stopping power of ions in FEG system very well [19]. However, the TCS approach has a very slow convergence of the results to the expected Bethe formula, while the IDA approach tends to overestimate the stopping values at low energies.

\section{B. Cluster stopping power}

Let us now consider two point charges $Z_{1}$ and $Z_{2}$, located at $\vec{r}_{1}$ and $\vec{r}_{2}$, and separated by $\vec{r}_{m}=\vec{r}_{2}-\vec{r}_{1}$ as shown in Fig. 1 . Therefore, the stopping force on the charge labeled 1 can be written as

$$
\left(\frac{d E}{d z}\right)_{1}=-\frac{1}{v}\left(\vec{F}_{\text {ind }}^{(1)}+\vec{F}_{\text {ind }}^{(2)}\right)_{\vec{r}=\vec{r}_{1}} \cdot \vec{v},
$$

where $F_{\text {ind }}^{(i)}$ is the induced force generated by the charge $i=$ 1,2 at the position of charge 1 . Here, we assume the two wake potentials are added linearly. Therefore, the simultaneous interaction of the scattered electrons with the two centers is neglected.

The second term from Eq. (6) is the main one responsible for the vicinage effect and can be calculated from induced 
density $\left(n_{\text {ind }}^{(2)}\right)$ generated by charge 2 as

$$
\begin{aligned}
\vec{F}_{\text {ind }}^{(2)}\left(\vec{r}=\vec{r}_{1}\right) & =Z_{1} \int \nabla_{\vec{r}_{1}}\left(\frac{n_{\text {ind }}^{(2)}\left(\vec{r}^{\prime}-\vec{r}_{2}\right)}{\left|\vec{r}_{1}-\vec{r}^{\prime}\right|}\right) d^{3} r^{\prime} \\
& =-Z_{1} \int \nabla_{\vec{r}_{m}}\left(\frac{n_{\text {ind }}^{(2)}\left(\vec{r}^{\prime \prime}\right)}{\left|\vec{r}_{m}+\vec{r}^{\prime \prime}\right|}\right) d^{3} r^{\prime \prime} .
\end{aligned}
$$

Since $\vec{r}_{m}$ is usually not determined experimentally, an average over all directions and thermal vibrations has to be performed. Let $P\left(\vec{r}_{m}\right)$ the probability density to find the fragments separated by $\vec{r}_{m}$. Using this probability density to average Eq. (7), we have

$$
\begin{aligned}
\left\langle\vec{F}_{\text {ind }}^{(2)}\right\rangle & =-Z_{1} \iint P\left(\vec{r}_{m}\right) \nabla_{\vec{r}_{m}}\left(\frac{n_{\text {ind }}^{(2)}\left(\vec{r}^{\prime \prime}\right)}{\left|\vec{r}_{m}+\vec{r}^{\prime \prime}\right|}\right) d^{3} r^{\prime \prime} d^{3} r_{m} \\
& =-Z_{1} \int\left(\nabla_{\vec{r}^{\prime \prime}} \Phi\left(\vec{r}^{\prime \prime}\right)\right) n_{\text {ind }}^{(2)}\left(\vec{r}^{\prime \prime}\right) d^{3} r^{\prime \prime},
\end{aligned}
$$

where

$$
\Phi(\vec{r})=\int \frac{P\left(\vec{r}_{m}\right)}{\left|\vec{r}+\vec{r}_{m}\right|} d^{3} r_{m} .
$$

Considering the probability density to find the fragment given by $P\left(\vec{r}_{m}\right)=\delta\left(r_{m}-r_{0}\right) /\left(4 \pi r_{0}^{2}\right)$, i.e, the companion can be found at any angle in a shell of radius $r_{0}$, if we neglect the radial distribution due to thermal vibrations. Under this condition, Eq. (9) yields the solution for the electrostatic potential produced by a spherical shell, namely $\Phi(r)=1 / r$ for $r>r_{0}$ and $1 / r_{0}$ otherwise and therefore Eqs. (8) and (6) can be written as

$$
\left(\frac{d E}{d z}\right)_{1}=\frac{d E}{d z}\left(Z_{1}\right)+I\left(Z_{1}, Z_{2}, r_{0}\right),
$$

where the first term is the direct stopping force given by Eq. (5) and the interference term $I\left(Z_{1}, Z_{2}, r_{0}\right)$ is the vicinage stopping force. It can be evaluated by inserting Eq. (2) written for $n_{\text {ind }}^{(2)}$ in Eqs. (8) and (9) using $\Phi^{\prime}(r)=-1 / r^{2}$ for $r>r_{0}$ and 0 otherwise as

$$
\begin{aligned}
& I\left(Z_{1}, Z_{2}, r_{0}\right) \\
& \quad=-Z_{1} \int \Theta\left(r^{\prime \prime}-r_{0}\right) \frac{n_{\text {ind }}^{(2)}\left(\vec{r}^{\prime \prime}\right)}{\left(r^{\prime \prime}\right)^{2}} \cos \theta^{\prime \prime} d^{3} r^{\prime \prime},
\end{aligned}
$$

resulting in

$$
\begin{aligned}
I\left(Z_{1}, Z_{2}, r_{0}\right)= & \frac{Z_{1}}{2 \pi v^{2}} \int_{\left|v_{F}-v\right|}^{v_{F}+v} d k \\
& \times\left(2 k^{2}\left(v_{F}^{2}+v^{2}\right)-k^{4}-\left(v_{F}^{2}-v^{2}\right)^{2}\right) \\
& \times \sum_{\ell=0}^{\infty}(\ell+1) \sin \left(\delta_{\ell}^{(2)}(k)-\delta_{\ell+1}^{(2)}(k)\right) \\
& \times \int_{r_{0}}^{\infty} d r \mathcal{R}_{k, \ell}^{(2)}(r) \mathcal{R}_{k, \ell+1}^{(2)}(r),
\end{aligned}
$$

after straightforward but cumbersome calculations as in [18]. The superscript (2) indicates the phase shifts $\delta_{\ell}$ and radial wave functions $\mathcal{R}_{k, \ell}(r)$ from the electron-ion potential, which is due to charge 2 . Of course the vicinage effect on charge 2 can be calculated just by changing the sub- and superscripts $1 \leftrightarrow 2$. It is easy to observe that for $r_{0} \rightarrow \infty$ the vicinage effect vanishes, and for $r_{0} \rightarrow 0$ the term $I\left(Z_{1}, Z_{2}, 0\right)=$ $\frac{Z_{1}}{Z_{2}} \frac{d E}{d z}\left(Z_{2}\right)$ [see Eq. (A1) from Ref. [18]]. Therefore, it gives the right united-atom limit for high-projectile energies since the total cluster stopping power,

$$
\begin{aligned}
\left(\frac{d E}{d z}\right)_{\text {united }}= & \left(\frac{d E}{d z}\right)_{1}+\left(\frac{d E}{d z}\right)_{2} \\
= & \frac{d E}{d z}\left(Z_{1}\right)+I\left(Z_{1}, Z_{2}, 0\right) \\
& +\frac{d E}{d z}\left(Z_{2}\right)+I\left(Z_{2}, Z_{1}, 0\right) \\
= & \left(Z_{1}+Z_{2}\right)^{2} \frac{\omega_{p}^{2}}{v^{2}} \ln \left(\frac{2 v^{2}}{\omega_{p}}\right),
\end{aligned}
$$

results in the Bethe formula for a FEG system described by the plasmon frequency $\omega_{p}$.

As demonstrated recently by IDA calculations [19], the best choice for the electron-ion potential is the Yukawa potential with inverse of screening length $\alpha$ obtained from a dynamical interpolation between the high-velocity solution $\alpha=\omega_{p} / v$ and the values for the static limit $\alpha_{0}$ as obtained by DFT with exchange-correlation terms. Such results have a rather good agreement with full TDDFT calculations for a wide range of projectile energies.

We can further improve the description of the electron-ion potential to allow for the influence of the companion fragment by using the following electron-ion potential

$$
V_{i}^{(j)}(r)=-\frac{Z_{i}+Q_{\text {ind }, \ell}^{(j)}}{r} \exp (-\alpha r),
$$

where $Q_{\mathrm{ind}, \ell}^{(j)}$ is the induced charge generated by the charge $j$ on the charge $i$, which provides a further screening for the electron-ion interaction. It depends on the angular momentum $\ell$ and is derived in Appendix A. It is pointed out that this screening will affect not only the second term of Eq. (10) but also the first one. This procedure is similar to the one described in Ref. [27] for the vicinage effect of the ion charge state. The present model described by Eq. (10) is called IDAMol in what follows.

\section{Linear theory}

Within the framework of the dielectric formalism the stopping power of ions in a electron gas system is given by the following expression [28]:

$$
\frac{d E}{d z}=\frac{2 Z_{1}^{2}}{\pi v^{2}} \int_{0}^{\infty} \frac{d q}{q} \int_{0}^{q v} d \omega \omega \operatorname{Im}\left[\frac{-1}{\epsilon(q, \omega)}\right],
$$

for an ionic point charge $Z_{1} . \epsilon(q, \omega)$ is the dielectric function, which for an electron-gas system was calculated by Lindhard and reads $\epsilon_{L}\left(q, \omega, \omega_{p}\right)$ (see, for instance, [29] for explicit expressions). For the cluster ions the stopping power is modified by the vicinage effect $I$ according to

$$
I=\frac{Z_{1} Z_{2}}{\pi v^{2}} \int_{0}^{\infty} \frac{d q}{q} \frac{\sin \left(q r_{0}\right)}{q r_{0}} \int_{0}^{q v} d \omega \omega \operatorname{Im}\left[\frac{-1}{\epsilon(q, \omega)}\right],
$$


for two neighboring point charges $Z_{1}$ and $Z_{2}$ distant by $r_{0}$, after averaging over all orientations. The use of Eq. (16) to describe different experimental data can be found in Ref. [5].

It is pointed out that the results from Eq. (5) in the perturbative limit of the IDA approach for a Yukawa potential are well described making the following replacement in Eq. (15):

$$
\operatorname{Im}\left[\frac{-1}{\epsilon(q, \omega)}\right] \rightarrow \frac{\operatorname{Im}\left[\epsilon_{L}(q, \omega)\right]}{1+(\alpha / q)^{2}}
$$

where $\alpha$ is the same used in Eq. (5) and is given by $\omega_{p} / v$ for $v \gg v_{f}$. Equation (17) is derived for a central electron-ion potential as the Yukawa potential. The same holds true for the vicinage effect $I$ in Eq. (12). It is also very well described in the perturbative IDA limit by the replacement of Eq. (17). The use of Eq. (17) yields the correct behavior for $v>v_{f}$.

\section{EXPERIMENTAL PROCEDURE}

The energy loss of $\mathrm{H}^{+}$ions after the breakup of incident $\mathrm{HeH}^{+}$ions has been measured in $\mathrm{Al}_{2} \mathrm{O}_{3}$ using the medium energy ion scattering (MEIS) technique as reported in Refs. [15,30-32] for $\mathrm{H}_{2}{ }^{+}$ions and other targets. In short, thin $\mathrm{Al}_{2} \mathrm{O}_{3}$ films with $3.5 \mathrm{~nm}$ of thickness were deposited above a carbon substrate via RF magnetron sputtering (AJA Orion-8) and mounted on a three-axis goniometer of the MEIS facility at the Ion Implantation Laboratory (LII) at UFRGS. The chamber containing the samples was connected to a 500$\mathrm{kV}$ electrostatic accelerator manufactured by HVEE, which provided a stable $\mathrm{H}^{+}$and $\mathrm{HeH}^{+}$beam with energies from 50 to $80 \mathrm{keV} / \mathrm{u}$, with currents between 5 and $20 \mathrm{nA}$ along the sample normal. The backscattered $\mathrm{H}^{+}$ions emerging from the target were analyzed using a toroidal electrostatic analyzer (TEA) mounted at 120 degrees with respect to the beam direction. The overall energy resolution of the system is about $400 \mathrm{eV}$ for $50-\mathrm{keV} \mathrm{H}^{+}$detected ions.

Two-dimensional (2D) maps of ion scattering intensities as a function of the detected energies and scattering angles (2D MEIS spectra) were measured and the energy spectra [onedimensional (1D) MEIS spectrum] at three scattering angles $\left(108^{\circ}, 120^{\circ}\right.$ and $\left.132^{\circ}\right)$ were analyzed. They were simulated by the POWERMEIS package $[33,34]$, which was extended to include vicinage and Coulomb explosion effects as described in Ref. [31]. Application of the MEIS technique at large backscattering angles means that the fragments are correlated only during the incoming path and to some extent during the backscattering collision. The dwell time for the incoming path inside the thin film is only about $1 \mathrm{fs}$, too short for any dynamic alignment of the projectile species. Thus we assume a random orientation.

\section{DISCUSSION}

The IDAMol is first checked for consistency against widely accepted and successful linear models by turning of its nonlinear effects for the comparison in Fig. 2. For this sake Eq. (12) was evaluated for a hypothetical small charge $Z_{1}=$ $Z_{2}=0.01$ and $\omega_{p}=0.5$ (corresponding to an electron-radius density parameter $\left.r_{s}=2.29\right)$ as a function of the internuclear distance $r_{0}$. The inverse of screening length was set to

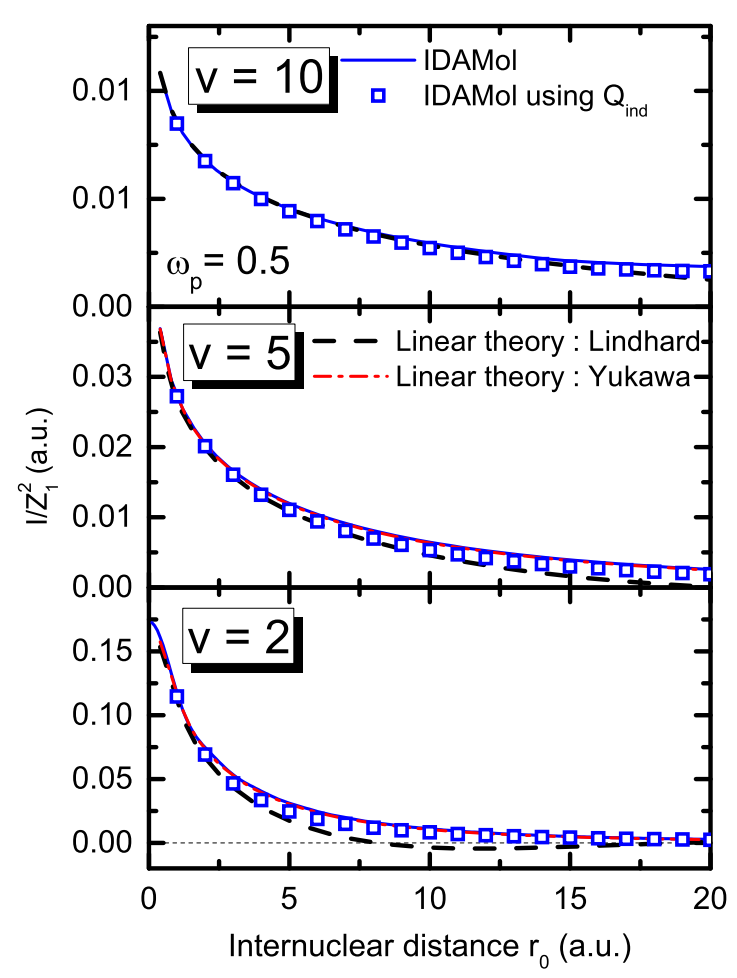

FIG. 2. Comparison of different models of the vicinage effect $I$ divided by $Z_{1}^{2}\left(Z_{1} \rightarrow 0\right)$ for a homonuclear dicluster as a function of the internuclear distance $r_{0}$ and different cluster velocities $v$. See text for the details of each model.

$\alpha=\omega_{p} / v$. The results correspond to the solid blue lines and coincide with results from the linear model given by the Yukawa approximation as described by Eq. (17) (the corresponding red dash-dotted lines are nearly identical to the solid blue curves). However, this linear model differs slightly from the formalism where the Lindhard dielectric function is used (called dielectric formalism [4,5]) (black dashed line) for large internuclear distances. In particular for $v=2$ the vicinage effect is negative at around $r_{0}=10$ whereas the present model gives always $I>0$ for the present cluster velocities. The reason for this disagreement is the effect of the induced charge generated by the companion fragment on the scattering potential. This effect was taken into account approximately by using Eq. (14) with $Q_{\text {ind, } \ell}^{(j)}$ values from Appendix A. It reduces the vicinage effect $I$ (see open squares) towards the results of the linear theory from dielectric formalism (black dashed lines). The IDAMol vicinage effect, however, stays positive (for $r_{0}<20$ ) when considering this correction.

Figure 3 shows the vicinage effect $I$ for different homonuclear diclusters separated by $r_{0}=2$. The results are normalized by $Z_{1}^{2}$ in order to merge all results at high cluster velocities. The results for $Z_{1}=0.01$ coincide with the ones determined from Eqs. (16) and (17) but disagree at small velocities with those of the dielectric formalism. The reason for this disagreement is the use of the central approximation for the electron-ion scattering [35]. Nonlinear effects decrease the vicinage effect and can even invert its sign as observed for $Z_{1} \geqslant 1$ at $v \approx 1$. The negative $I$ values arise from a shift and/or compaction of the electronic screening cloud 


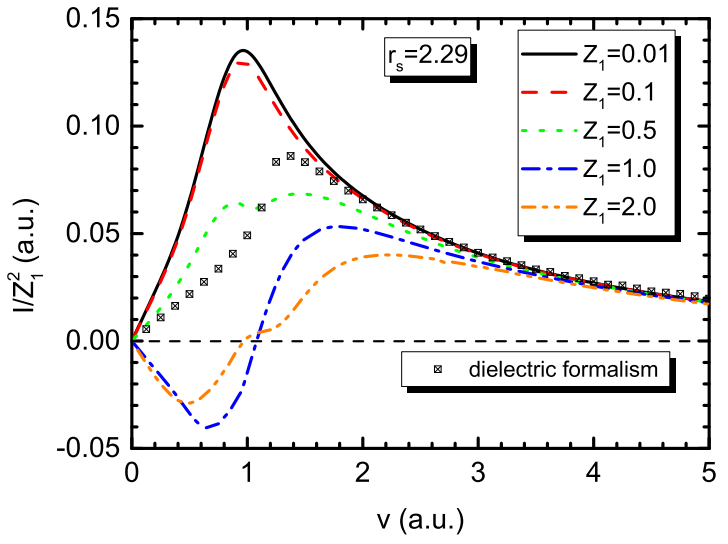

FIG. 3. The vicinage effect $I$ divided by $Z_{1}^{2}$ for a homonuclear dicluster separated by $r_{0}=2$ as a function of the velocity $v$ and charge $Z_{1}$. Strong nonlinear effects are observed for $Z_{1} \gg 0.1$ and low velocities $v<3$.

behind the leading ion. The center position and shape of this cloud have a strong influence on the dynamics of the trailing companion ion. A high induced electron density in front of the trailing ion may accelerate this ion, leading to a significant reduction of the $I$ value even below 0 . The $Z_{1}$ dependence of the scaled $I$ value in Fig. 3 is a clear proof of nonlinear effects. The main variation of these curves seems to appear around $Z_{1}=0.5$ and there is not much difference between the results for $Z_{1}=1$ and $Z_{1}=2$. This behavior is an indication for the influence of some higher orders of the interaction.

Furthermore, it is pointed out that negative $I$ values can also be obtained in linear theory. One example is given by the dieletric formalism at large internuclear distances (see Fig. 2). Another example is connected to bound-electron systems, as demonstrated in Ref. [14] for an oscillator model. Nevertheless, the latter model could not explain the magnitude and energy position of negative $I$ values observed in the experimental data.

The IDAMol results are compared in Fig. 4 to $\mathrm{H}_{2}{ }^{+}$experimental data for the stopping ratio $\mathrm{R}_{\mathrm{H}^{+}}$defined as

$$
\mathrm{R}_{\mathrm{H}^{+}}=\left(\frac{d E}{d z}\left(\mathrm{H}^{+}\right)\right)^{\text {cluster }} /\left(\frac{d E}{d z}\left(\mathrm{H}^{+}\right)\right)^{\text {indep. }},
$$

where $\left(d E / d z\left(\mathrm{H}^{+}\right)\right)^{\text {cluster }}$ is the stopping power of $\mathrm{H}^{+}$ surrounding by its companion $\left(\mathrm{H}^{+}\right.$or $\mathrm{H}^{0}$ ions $)$ and $\left(d E / d z\left(\mathrm{H}^{+}\right)\right)^{\text {indep. }}$ is the stopping power for independent $\mathrm{H}^{+}$ ions (no vicinage effect). The stopping ratios were measured for ultrathin $\mathrm{SiO}_{2}$ and $\mathrm{Al}_{2} \mathrm{O}_{3}$ films as a function of the incident $\mathrm{H}_{2}{ }^{+}$energy [15,32] and therefore the distance between the fragments did not change much during the dwell time. In addition, both films have similar stopping ratio $\mathrm{R}_{\mathrm{H}^{+}}$because they have similar electronic densities of the valence electrons.

Since the present formalism was developed for a freeelectron gas system, it has to be used with some caution for the present oxides, although their energy loss functions (ELF) have just one peak, which can be modeled as a plasmon peak from a free-electron model. Data [36] and atomic coupledchannel calculations [37] for $\mathrm{H}+\mathrm{He}$ deviate by more than a factor 4 from the simple velocity scaling of a free-electron

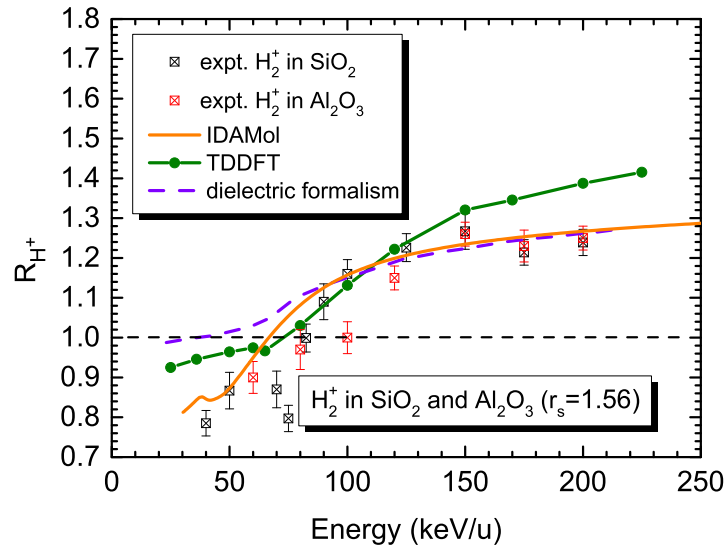

FIG. 4. Stopping-power ratio for $\mathrm{H}^{+}$ions as a function of the $\mathrm{H}_{2}{ }^{+}$incident energy in ultrathin $\mathrm{SiO}_{2}$ and $\mathrm{Al}_{2} \mathrm{O}_{3}$ films. The symbols are the experimental data from $[15,32]$ and the lines correspond to different models: dielectric formalism (violet dashed line), TDDFT calculations [32] (green line), and present work IDAMol (orange solid line).

gas, at $v=0.35$. However, according to Ref. [38], a Gd-oxide surface layer does not give rise to significant deviations from such a velocity proportionality of proton stopping even down to $v=0.15$. This is in accord with other searches for such deviations in oxides [39], where only a faint influence of a gap might be visible at proton energies above 2 or $3 \mathrm{keV}$. Thus, it seems that either strong perturbations or also quasimolecular effects might open many electron-reaction channels that allow for a quasifree electronic motion during atomic interaction processes. Furthermore, it is noted that even for $\mathrm{H}+\mathrm{He}$, deviations become visible only at energies below about $15 \mathrm{keV} / \mathrm{u}$ $(v=0.8)$. This means, we expect no significant influence of target bound-state effects for the current experimental data at $\geqslant 40 \mathrm{keV} / \mathrm{u}$. Alternatively, we could have used noble metals to prevent the issue of oxidation. However, such noble metals may not be described as a simple FEG, since they involve a complicated ELF at the optical limit. Oxides are consequently used in the experiments and calculations with the present model reproduce the experimental data reasonably well. Nevertheless, it is still not clear if the agreement is a general phenomenon, since the present model neglects possible effects of strong covalent bonding and corresponding changes in the electron density.

For the theoretical models displayed in Fig. 4 we have used then a single value of density parameter $r_{s}=1.56$ to describe both films.

As can be observed in Fig. 4, the stopping ratios measured for $\mathrm{SiO}_{2}$ and $\mathrm{Al}_{2} \mathrm{O}_{3}$ change from values smaller than one, of about 0.8 at low $\mathrm{H}_{2}{ }^{+}$energies to values larger than one, of about 1.3 at high energies. In fact, all theory curves as well as the experimental data are flattening towards the lowest energy. This is the velocity range, where details of the band structure become more and more important. The crossing of unity appears at about $83 \mathrm{keV} / \mathrm{u}$ in the experimental data set. In contrast, this switching from negative to positive vicinage effects is shifted down to $38 \mathrm{keV} / \mathrm{u}$ for the dielectric formalism. Indeed TDDFT calculations performed recently for an electron gas system with the same density parameter 


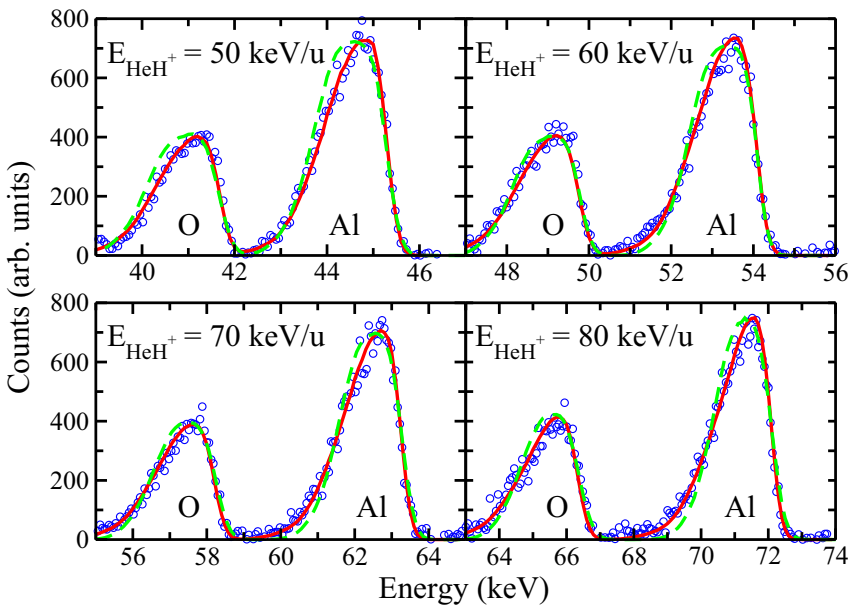

FIG. 5. Experimental 1D MEIS energy spectra (open blue circles) for $\mathrm{HeH}^{+}$projectiles on a thin $\mathrm{Al}_{2} \mathrm{O}_{3}$ film for different cluster energies and a scattering angle of $128^{\circ}$. The $\mathrm{H}^{+}$ions from the breakup are detected after backscattering off $\mathrm{Al}$ and $\mathrm{O}$. The solid and dashed lines (red and green, respectively) correspond to POWERMEIS simulations for $\mathrm{HeH}^{+}$and $\mathrm{H}^{+}$projectiles, respectively.

$r_{s}=1.56$ show the importance of higher-order terms for the description of negative vicinage effects at low energies (see green line) and of the switching point. Nevertheless, these calculations are still not able to reproduce the experimental data at the lowest energies and seem to fail to converge to the dielectric results at asymptotically high energies. On the other hand, the present IDAMol calculations agree with experimental data below $63 \mathrm{keV} / \mathrm{u}$ much better and converge to the dielectric results at high projectile energies. At high energies in the united-atom (UA) limit (at $r_{0}=0$ ) the vicinity effect should approach $R_{\mathrm{UA}}=2$ for homonuclear systems. At finite $r_{0}$ values, a gradual variation of interference effects occurs [4] and average values of $R=1.4 \ldots 1.5$ are obtained.

Finally, the vicinage effect on the stopping power of $\mathrm{H}^{+}$ ions perturbed by $\mathrm{He}$ for incident $\mathrm{HeH}^{+}$cluster ions were measured by MEIS. For this case higher-order effects should be much larger. The corresponding 1D MEIS spectra for the scattering angle of $128^{\circ}$ are shown in Fig. 5 for 50-, 60-, $70-$, and $80-\mathrm{keV} / \mathrm{u} \mathrm{HeH}^{+}$projectiles. Two peaks are visible and these are due to $\mathrm{H}^{+}$backscattering at $\mathrm{Al}$ and $\mathrm{O}$ atoms of the $\mathrm{Al}_{2} \mathrm{O}_{3}$ film. A carbon signal from the substrate was also measured at lower energies but not shown in Fig. 5. The energy spectra were fitted by the POWERMEIS software to get the vicinage and Coulomb explosion effects along the incoming trajectory before the backscattering collision. The energy spectra for incident $\mathrm{H}^{+}$ions at the same $\mathrm{HeH}^{+}$energy per nucleon were also measured.

For clarity only the corresponding full simulations are shown in Fig. 5 (green dashed line). Energy loss broadening due to Coulomb explosion [10] is clearly visible in both peaks at $80 \mathrm{keV} / \mathrm{u}$. A negative vicinage effect of the stopping power can be also identified at $50 \mathrm{keV} / \mathrm{u}$ since the widths of the peaks for $\mathrm{H}^{+}$projectiles are somewhat wider.

The results for the vicinage effect for incident $\mathrm{HeH}^{+}$ projectiles, namely the ratio of stopping power of $\mathrm{H}^{+}$ions traveling with the He ions and the stopping power of uncorre-

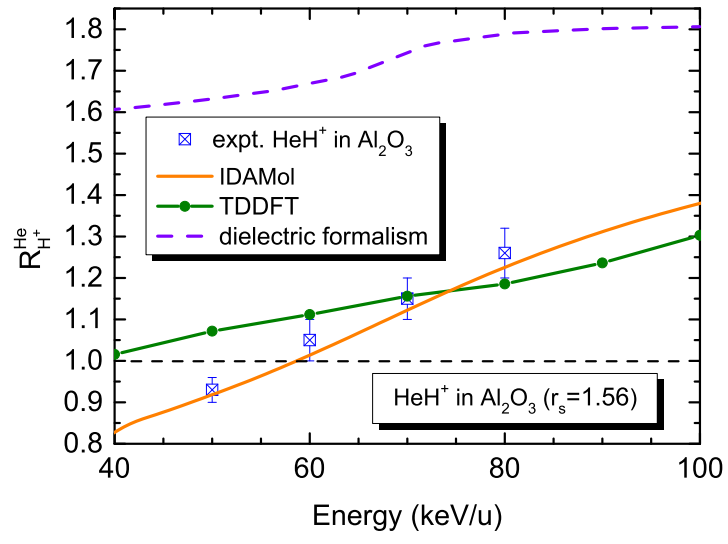

FIG. 6. Experimental results for the stopping-power ratio of $\mathrm{H}^{+}$ ions in $\mathrm{Al}_{2} \mathrm{O}_{3}$ for incident $\mathrm{HeH}^{+}$projectiles in comparison with recent calculations using the present IDAMol model (orange solid line) and TDDFT (green line).

lated $\mathrm{H}^{+}$ions is displayed in Fig. 6. The IDAMol (orange solid line) can describe the present measurements very well, staying within the error bars of the four experimental data points. In addition strong nonlinear effects are identified by comparing with linear results from dielectric formalism (see violet dashed line). Linear predictions calculated using Eqs. (19) and (20) are in strong disagreement with experimental data by almost a factor of 2 at low energies for $\mathrm{He}$ as a companion ion. Thus, linear interaction models should overestimate the stopping-power ratio in Fig. 6 more drastically than in Fig. 4. Furthermore, in Fig. 6 the nonlinear models agree better with the experimental data. This shows that a reasonable description of the nonlinear dynamics is more important than band-structure details for higher charges. TDDFT calculations were also performed for $r_{s}=1.56$. The same methodology as of Ref. [32] was applied to the $\mathrm{HeH}^{+}$dimer. The distance between $\mathrm{He}^{2+}$ and $\mathrm{H}^{+}$was set to 1.46 a.u. and was fixed during time propagation. We considered three different orientations of the dimer, namely two parallel $\left(\mathrm{He}^{2+}\right.$ follows $\mathrm{H}^{+}$(par, 21) or $\mathrm{H}^{+}$follows $\mathrm{He}^{2+}$ (par, 12) along the pass through the target) and one perpendicular orientation [the two ions are perpendicular to the direction of motion with $\mathrm{He}^{2+}$ below $\mathrm{H}^{+}$(per,21)]. The stopping ratio of the hydrogen ion was calculated using the following expression:

$$
R_{\mathrm{H}^{+}}^{\mathrm{He}}=1+\left[\frac{\left(R_{\mathrm{dim}}-1\right)}{2}\right]\left[1+\left(\frac{S_{\mathrm{He}^{2+}}}{S_{\mathrm{H}^{+}}}\right)\right],
$$

where $S_{\mathrm{He}^{2+}}$ and $S_{\mathrm{H}^{+}}$are the stopping powers of independent $\mathrm{He}$ and $\mathrm{H}$ ions and $R_{\mathrm{dim}}$ is the total stopping power of the dimer relative to the sum of independent components:

$$
R_{\mathrm{dim}}=\frac{S_{\mathrm{dim}}^{\mathrm{av}}}{\left(S_{\mathrm{He}^{2+}}+S_{\mathrm{H}^{+}}\right)} .
$$

The total stopping power of the dimer is averaged as (see Appendix B) [40]

$$
S_{\mathrm{dim}}^{\mathrm{av}}=\frac{1}{3}\left[2 S_{\mathrm{dim}}^{\mathrm{per}, 21}+\frac{1}{2}\left(S_{\mathrm{dim}}^{\mathrm{par}, 21}+S_{\mathrm{dim}}^{\mathrm{par}, 12}\right)\right] .
$$

The TDDFT result (green line) agrees much better with the experimental data than the linear theory, but it overestimates 
the vicinage effect at low energies, similar to Fig. 4. A possible reason for this disagreement could be related to the averaging procedure justified only in the linear regime as derived in the Appendix B. In fact TDDFT are time-consuming calculations and thus we did not properly average over all possible orientations of the dimer (as opposed to the IDAMol results).

\section{CONCLUSIONS}

A nonperturbative model for the vicinage effect on the stopping power of cluster ions in matter (IDAMol) was developed by generalizing the stopping-power formula (IDA model) derived recently $[18,19]$ for ions slowing down in an electron-gas system. For this sake it was assumed that the cluster orientation is not aligned to any particular direction and a general expression Eq. (12) was derived in terms of the phase shifts and radial-wave functions of the electron-ion collision. This expression for the vicinage effects reproduces the results of the dielectric formalism at high cluster energies (for $\mathrm{H}_{2}{ }^{+}$) and gives strong nonlinear effects (for $\mathrm{HeH}^{+}$), which lead to a good agreement with the experimental data for the stopping-power ratio of incident $\mathrm{HeH}^{+}$ions in ultrathin $\mathrm{Al}_{2} \mathrm{O}_{3}$. For the existing experimental data of $\mathrm{H}_{2}{ }^{+}$in $\mathrm{SiO}_{2}$ and $\mathrm{Al}_{2} \mathrm{O}_{3}$ and $\mathrm{HeH}^{+}$in $\mathrm{Al}_{2} \mathrm{O}_{3}$ we find a good agreement with the IDAMol calculations over an extended range of energies (Figs. 4 and 6), explaining the observed transition from positive to negative vicinage effects at lower energies. From all these finding we may conclude that IDAMol has an extremely large range of validity concerning projectile charge as well as projectile speed.

\section{ACKNOWLEDGMENTS}

We are indebted to the Brazilian agencies $\mathrm{CNPq}$ and FAPERGS for the partial support of this research project. This study was financed in part by the Coordenação de Aperfeiçoamento de Pessoal de Nível Superior-Brasil (CAPES)Finance Code 001. One of the authors (F.M.) acknowledges the Brazilian fellowship from CNPq. F.M. and N.E.K. acknowledge financial support by the Departamento de Educación, Universidades e Investigación, the University of the Basque Country UPV/EHU (Grant No. IT-756-13) and the Spanish Ministerio de Economía y Competitividad (Grants No. FIS2013-48286-C02-02-P and No. FIS2016-76471-P). We are grateful to Dr. A. G. Borisov for the permission to use the TDDFT propagation code developed by him. We thank Dr. R. Díez Muiño for helpful discussions on the manuscript.

\section{APPENDIX A: CORRECTION FOR THE POLARIZATION CHARGE IN THE SCATTERING POTENTIALS DUE TO DIMER-ELECTRON INTERACTION}

In the ion-target interaction, the incident electron sees a scattering potential screened by the electrons in the medium. Let us assume that the interaction process is one in which the electron scattering occurs with the $Z_{1}$ ion. Depending on the value of the orbital angular momentum of the incident electron, the electron sees a screened potential whose screening charge is a mixture of polarization charges induced from $Z_{1}$ and $Z_{2}$ ions. Then, it is necessary to know what is the polarization charge created by the $Z_{2}$ ion, since, depending on the impact parameter at which the electron undergoes scattering, this polarization charge must be added to the polarization charge induced by $Z_{1}$. The induced charges at both scattering centers can be determined by integration of the induced charge density. Then, the induced charges in the $Z_{j}$ position can be written as

$$
Q_{\text {ind }, \ell}^{(j)}=\int_{0}^{r^{*}} d r r^{2} \int d \Omega \rho_{\text {ind }, \ell}^{(j)} .
$$

The upper limit $r^{*}$ is written by using the semiclassical approach as a function of $\ell$ and $k$ and is given by

$$
r^{*} \approx \mathcal{A} \frac{(\ell+1 / 2)}{k},
$$

where the ad hoc factor $\mathcal{A}$ assumes the value $\mathcal{A}=1.5$ for the $\mathrm{H}_{2}^{2+}$ dimer. The term $(\ell+1 / 2)$ corresponds to eigenvalues of the semiclassical angular momentum operator $L$. Depending on the value of $r^{*}$, the induced charge density around one of the scattering centers can be modified by the other center. This effect influences directly the projectile's energy loss.

According to Eq. (A1), it is noted the integral over the whole volume is equal to the $j$ th charge (that is, $-Z_{j}$ ), but here we are interested only in the fraction of that charge that will influence the scattering of the electrons with the charge $i$ (with $i \neq j$ ). The induced charge density $\rho_{\text {ind } \ell}^{(j)}$ is calculated by performing an angular averaging on $\vec{r}_{0}$ through the Poisson's equation, that is,

$$
\rho_{\text {ind }, \ell}^{(j)}\left(r_{a}\right)=-\frac{1}{4 \pi} \nabla_{\vec{r}}^{2} V_{\text {ind }, \ell}^{(j)}\left(r_{a}\right) .
$$

where $\vec{r}_{a} \equiv \vec{r}-\vec{r}_{M}$. The induced potential is taken from the difference between the potentials of Yukawa and Coulomb:

$$
V_{\text {ind, } \ell}^{(j)}\left(r_{a}\right)=-Z_{j}\left(\frac{e^{-\alpha\left|r_{a}\right|}}{\left|r_{a}\right|}-\frac{1}{\left|r_{a}\right|}\right),
$$

$V_{\text {ind }, \ell}^{(j)}\left(r_{a}\right)$ can be written in terms of the multipole expansion, and thus using Eq. (A3) we obtain

$$
\rho_{\text {ind }, \ell}^{(j)}\left(r_{<}, r_{>}\right)=-\frac{Z_{j}}{4 \pi} \nabla_{r}^{2}\left(\frac{\sinh \left(\alpha r_{<}\right)}{\alpha r_{<}} \frac{e^{-\alpha r_{>}}}{r_{>}}-\frac{1}{r_{>}}\right),
$$

where $r_{<}=\min \left(r, r_{0}\right)$ and $r_{>}=\max \left(r, r_{0}\right)$.

Finally from Eqs. (A1) and (A5), the induced charge $Q_{\text {ind, } \ell}^{(j)}$ can be obtained:

$$
\begin{aligned}
Q_{\text {ind }, \ell}^{(j)}= & -\frac{Z_{j} \alpha}{4 \pi r_{0}} \int_{0}^{r^{*}} d r r\left[e^{-\alpha r_{0}} \sinh (\alpha r) \Theta\left(r_{b}\right)\right. \\
& \left.+e^{-\alpha r} \sinh \left(\alpha r_{0}\right) \Theta\left(r_{a}\right)\right],
\end{aligned}
$$

where $\Theta(x)$ is the Heaviside step function, $r_{a} \equiv r-r_{0}$, and $r_{b} \equiv r_{0}-r$. As shown in Fig. 2, the use of $Q_{\text {ind }}$ is a linear correction for the vicinage effect I (see open squares).

\section{APPENDIX B: DIELECTRIC FORMULATION FOR THE TERMS OF PERPENDICULAR AND PARALLEL INTERFERENCE}

For two charges $Z_{1} e$ and $Z_{2} e$ in correlated motion with velocity $\vec{v}$ and internuclear separation $\vec{r}_{0}\left(\vec{r}_{0}=\vec{r}_{1}-\vec{r}_{2}\right)$, the 
energy loss of both charges is given by [4]

$$
\begin{aligned}
\frac{d E}{d z}= & \frac{1}{2 \pi^{2} v} \int d^{3} k \frac{\vec{k} \cdot \vec{v}}{k^{2}} \operatorname{Im}\left(-\frac{1}{\varepsilon(k, \omega)}\right) \\
& \times\left[\left(Z_{1}^{2}+Z_{2}^{2}\right)+2 Z_{1} Z_{2} \cos \left(\vec{k} \cdot \vec{r}_{0}\right)\right]
\end{aligned}
$$

Doing

$$
\int d^{3} k \rightarrow 4 \pi \int k^{2} d k \int_{0}^{\pi} \frac{\sin \theta_{k} d \theta_{k}}{2} \int_{0}^{2 \pi} \frac{d \varphi_{k}}{2 \pi}
$$

and using the variable $\omega=\vec{k} \cdot \vec{v}=k v \cos \theta_{k}$, we have $\sin \theta_{k} d \theta_{k}=-d\left(\cos \theta_{k}\right)=-d \omega / k v$, and thus we obtain

$$
\begin{aligned}
\frac{d E}{d z}= & \frac{2}{\pi v} \int_{0}^{\infty} k^{2} d k \int_{-k v}^{k v} \frac{1}{2} \frac{d \omega}{k v} \frac{\omega}{k^{2}} \operatorname{Im}\left(-\frac{1}{\varepsilon(k, \omega)}\right) \\
& \times \int_{0}^{2 \pi} \frac{d \varphi_{k}}{2 \pi}\left[\left(Z_{1}^{2}+Z_{2}^{2}\right)+2 Z_{1} Z_{2} \cos \left(\vec{k} \cdot \vec{r}_{0}\right)\right]
\end{aligned}
$$

\section{Interference term calculation: average on dimer orientations}

Taking $v \| \hat{z}$, and considering that $\theta_{k}$ is the angle between $\vec{k}$ and $\hat{z}, \theta_{0}$ is the angle between $\vec{r}_{0}$ and $\hat{z}$, and $\varphi^{\prime}=\varphi_{k}-\varphi_{0}$ is the angle that is made in the $x y$ plane between the projections of $\vec{r}_{0}$ and $\vec{k}$, we can define the interference term for average on

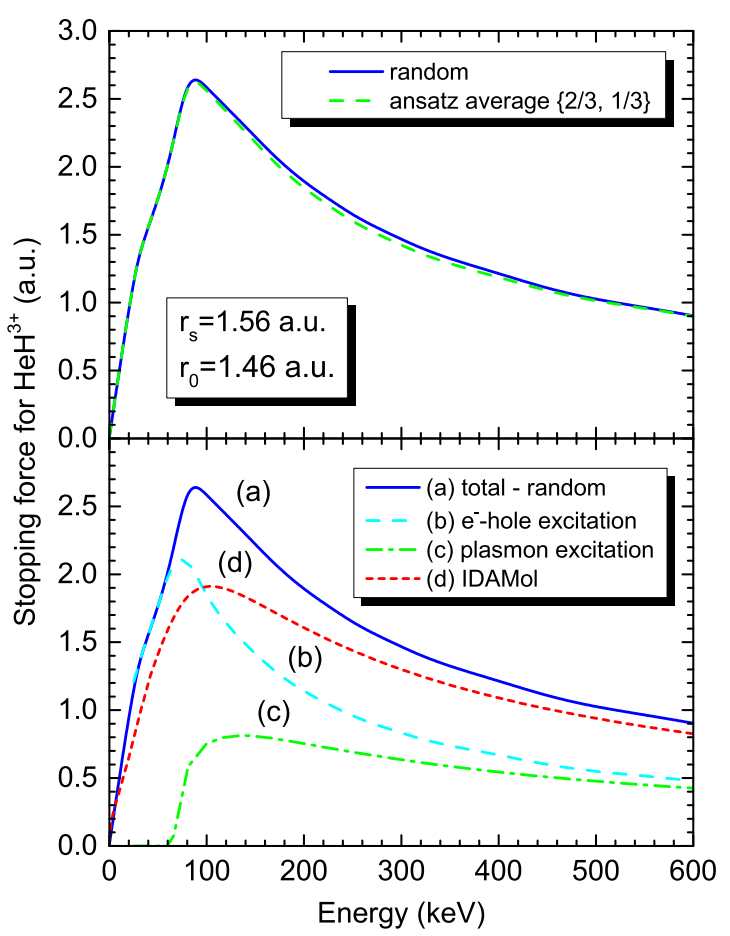

FIG. 7. Stopping force results of $\mathrm{HeH}^{3+}$ in $\mathrm{Al}_{2} \mathrm{O}_{3}\left(r_{s}=1.56\right)$ calculated from Lindhard formalism. (Upper panel) Blue solid line for random orientation of the dimer [Eq. (B9)] and green dashed line [Eq. (B10)] for the ansatz average $\{2 / 3,1 / 3\}$; (lower panel) contributions of the electron-hole and plasmon excitation to the energy loss of the dimer and the results from the IDAMol approach. dimer orientation as

$$
\begin{aligned}
\langle I\rangle_{\varphi}= & \int_{0}^{2 \pi} \frac{d \varphi_{k}}{2 \pi} \cos \left(\vec{k} \cdot \vec{r}_{0}\right) \\
= & \int_{0}^{2 \pi} \frac{d \varphi_{k}}{2 \pi} \cos \left[k r _ { 0 } \left(\cos \theta_{k} \cos \theta_{0}\right.\right. \\
& \left.\left.+\sin \theta_{k} \sin \theta_{0} \cos \varphi^{\prime}\right)\right]
\end{aligned}
$$

We consider now particular cases.

(1) Parallel orientation $\left(\vec{r}_{0} \| \vec{v}\right)$ :

$$
\left\langle I_{\|}\right\rangle_{\varphi}=\int_{0}^{2 \pi} \frac{d \varphi^{\prime}}{2 \pi} \cos \left(k r_{0} \cos \theta_{k}\right)=\cos \left(\frac{\omega r_{0}}{v}\right) .
$$

(2) Perpendicular orientation $\left(\vec{r}_{0} \perp \vec{v}\right)$ :

$$
\begin{aligned}
\left\langle I_{\perp}\right\rangle_{\varphi} & =\int_{0}^{2 \pi} \frac{d \varphi^{\prime}}{2 \pi} \cos \left(k r_{0} \sin \theta_{k} \cos \varphi^{\prime}\right) \\
& =J_{0}\left(k r_{0} \sqrt{1-\left(\frac{\omega}{k v}\right)^{2}}\right) .
\end{aligned}
$$

(3) Average over $\hat{r}_{0}$ (random orientation):

$$
\langle I\rangle_{\hat{r}_{0}}=\int_{0}^{\pi} \sin \theta_{0} d \theta_{0} \int_{0}^{2 \pi} d \varphi_{0} \cos \left(\vec{k} \cdot \vec{r}_{0}\right)=\frac{\sin k r_{0}}{k r_{0}} .
$$

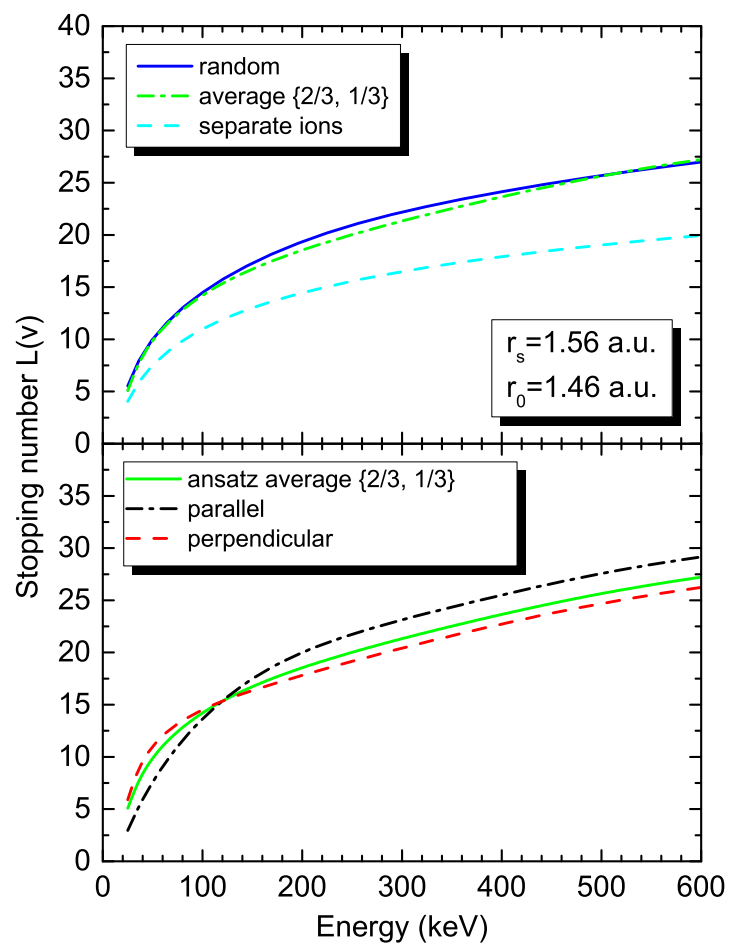

FIG. 8. (Upper panel) Stopping number results of $\mathrm{HeH}^{3+}$ in $\mathrm{Al}_{2} \mathrm{O}_{3} \quad\left(r_{s}=1.56\right)$. Here, calculations were performed using the plasmon-pole approximation to Lindhard dielectric function [41,42]. (Lower panel) The same as in upper panel, but now we have the energy loss contributions for the parallel and perpendicular orientations. 
(4) Ansatz average $\{2 / 3,1 / 3\}$. From Eqs. (B5) and (B6) we define

$$
I_{\mathrm{av}}=\frac{2}{3} J_{0}\left(k r_{0} \sqrt{1-\left(\frac{\omega}{k v}\right)^{2}}\right)+\frac{1}{3} \cos \left(\frac{\omega r_{0}}{v}\right) .
$$

Equation (B3) can now be written as

$$
\begin{aligned}
\left\langle\frac{d E}{d z}\right\rangle_{\text {rand }}= & \frac{1}{\pi v^{2}} \int \frac{d k}{k} \int_{-k v}^{k v} \omega d \omega \operatorname{Im}\left(-\frac{1}{\varepsilon(k, \omega)}\right) \\
& \times\left[\left(Z_{1}^{2}+Z_{2}^{2}\right)+2 Z_{1} Z_{2}\langle I\rangle_{\hat{r}_{0}}\right] .
\end{aligned}
$$

And, on the other hand, using the ansatz average we can define

$$
\begin{aligned}
\left\langle\frac{d E}{d z}\right\rangle_{\text {ansatz }}= & \frac{1}{\pi v^{2}} \int \frac{d k}{k} \int_{-k v}^{k v} \omega d \omega \operatorname{Im}\left(-\frac{1}{\varepsilon(k, \omega)}\right) \\
& \times\left[\left(Z_{1}^{2}+Z_{2}^{2}\right)+2 Z_{1} Z_{2}\langle I\rangle_{\mathrm{av}}\right] .
\end{aligned}
$$

Using Lindhard's dielectric function we have performed a full numerical calculation of Eqs. (B9) and (B10), and the results are shown in Fig. 7. Notice that in doing this integration we have included both collective and individual excitation of the electron gas. As can be seen in Fig. 7, the ansatz average of the Eqs. (B9) and (B10) provides an excellent approximation to the exact random average on the whole range of energies. Therefore, the average $\{2 / 3,1 / 3\}$ used in Eq. (21) to calculate the total stopping power of the dimer is very well justified.

In the lower panel of Fig. 7 we show the separate contributions to the total stopping corresponding to electron-hole excitations and to plasmon excitations [4] and we compare the total stopping force calculated with the dielectric function with the nonlinear IDAMol calculation described in Sec. II B.

Finally, in Fig. 8 (upper panel) we show the values of the so-called stopping number $[41,42]$, comparing the exact random average with the $\{2 / 3,1 / 3\}$ ansatz average and with the stopping number corresponding to separate ions, whereas in the lower panel we show the separate cases of parallel and perpendicular orientations and the $\{2 / 3,1 / 3\}$ average obtained from them. As it may be observed, there is a rather weak dependence on the orientations of the internuclear axis.
[1] W. Brandt, A. Ratkowski, and R. H. Ritchie, Phys. Rev. Lett. 33, 1325 (1974).

[2] J. Tape, W. Gibson, J. Remillieux, R. Laubert, and H. Wegner, Nucl. Instrum. Methods 132, 75 (1976).

[3] A. R. Nyaiesh, W. Steckelmacher, and M. W. Lucas, J. Phys. C 11, 2917 (1978).

[4] N. R. Arista, Phys. Rev. B 18, 1 (1978).

[5] N. R. Arista, Nucl. Instrum. Methods Phys. Res., Sect. B 164165, 108 (2000).

[6] R. Garcia-Molina, C. D. Denton, I. Abril, and N. R. Arista, Phys. Rev. A 62, 012901 (2000).

[7] Z. L. Mišković, S. G. Davison, F. O. Goodman, W.-K. Liu, and Y.-N. Wang, Phys. Rev. A 63, 022901 (2001).

[8] R. C. Fadanelli, P. L. Grande, and G. Schiwietz, Phys. Rev. A 77, 032902 (2008).

[9] N. Tahir, D. Hoffmann, J. Maruhn, and C. Deutsch, Nucl. Instrum. Methods Phys. Res., Sect. B 88, 127 (1994).

[10] S. M. Shubeita, R. C. Fadanelli, J. F. Dias, and P. L. Grande, Surface Science 608, 292 (2013).

[11] M. Quijada, A. G. Borisov, I. Nagy, R. Díez Muiño, and P. M. Echenique, Phys. Rev. A 75, 042902 (2007).

[12] J. M. Pruneda, D. Sánchez-Portal, A. Arnau, J. I. Juaristi, and E. Artacho, Phys. Rev. Lett. 99, 235501 (2007).

[13] N. E. Koval, D. Sánchez-Portal, A. G. Borisov, and R. Díez Muiño, Nucl. Instrum. Methods Phys. Res., Sect. B 317, 56 (2013).

[14] P. Sigmund and A. Schinner, Eur. Phys. J. D 61, 39 (2011).

[15] S. M. Shubeita, M. A. Sortica, P. L. Grande, J. F. Dias, and N. R. Arista, Phys. Rev. B 77, 115327 (2008).

[16] R. Levi-Setti, K. Lam, and T. R. Fox, Nucl. Instrum. Methods Phys. Res. 194, 281 (1982).

[17] J. C. Eckardt, G. Lantschner, N. R. Arista, and R. A. Baragiola, J. Phys. C: Solid State Phys. 11, L851 (1978).

[18] P. L. Grande, Phys. Rev. A 94, 042704 (2016).
[19] F. Matias, R. C. Fadanelli, P. L. Grande, N. E. Koval, R. Díez Muiño, A. G. Borisov, N. R. Arista, and G. Schiwietz, J. Phys. B 50, 185201 (2017).

[20] J. Finnemann, Master's thesis, Aarhus University, 1968.

[21] J. S. Briggs and A. P. Pathak, J. Phys. C 6, L153 (1973).

[22] P. Sigmund, Particle Penetration and Radiation Effects, Penetration of Atomic and Molecular Ions (Springer, Berlin, 2014), Vol. 2.

[23] H. B. Nersisyan, J. M. Fernandez-Varea, and N. R. Arista, Nucl. Instrum. Methods Phys. Res., Sect. B 354, 167 (2015).

[24] E. Bonderup, Institute of Physics, Aarhus, 1981 [http://www.phys.au.dk/-pl2X-sim-ahs/EBnotes.htm].

[25] A. F. Lifschitz and N. R. Arista, Phys. Rev. A 57, 200 (1998).

[26] A. Messiah, Quantum Mechanics, Vol. 2 (Dover, New York, 1963).

[27] S. Heredia-Avalos and R. Garcia-Molina, Phys. Rev. A 76, 032902 (2007).

[28] J. Lindhard, Mat. Fys. Medd. Dan. Vid. Selsk. 28, 1 (1954).

[29] P. Sigmund, Particle Penetration and Radiation Effects, General Aspects and Stopping of Swift Point Charges (Springer, Berlin, 2006), Vol. 1.

[30] S. M. Shubeita, P. L. Grande, J. F. Dias, R. Garcia-Molina, C. D. Denton, and I. Abril, Phys. Rev. B 83, 245423 (2011).

[31] L. F. S. Rosa, P. L. Grande, J. F. Dias, R. C. Fadanelli, and M. Vos, Phys. Rev. A 91, 042704 (2015).

[32] N. E. Koval, A. G. Borisov, L. F. S. Rosa, E. M. Stori, J. F. Dias, P. L. Grande, D. Sánchez-Portal, and R. D. Muiño, Phys. Rev. A 95, 062707 (2017).

[33] M. de Albuquerque Sortica, P. Grande, G. Machado, and L. Miotti, J. Appl. Phys. 106, 114320 (2009).

[34] G. Marmitt, Ph.D thesis, Universidade Federal do Rio Grande do Sul, 2017.

[35] A. Salin, A. Arnau, P. M. Echenique, and E. Zaremba, Phys. Rev. B 59, 2537 (1999).

[36] R. Golser and D. Semrad, Phys. Rev. Lett. 66, 1831 (1991).

[37] P. L. Grande and G. Schiwietz, Phys. Rev. A 47, 1119 (1993). 
[38] D. Roth, B. Bruckner, M. Moro, S. Gruber, D. Goebl, J. Juaristi, M. Alducin, R. Steinberger, J. Duchoslav, D. Primetzhofer et al., Phys. Rev. Lett. 118, 103401 (2017).

[39] D. Roth, B. Bruckner, G. Undeutsch, V. Paneta, A. I. Mardare, C. L. McGahan, M. Dosmailov, J. I. Juaristi, M. Alducin, J. D. Pedarnig et al., Phys. Rev. Lett. 119, 163401 (2017).
[40] H. M. Urbassek, V. Dröge, and R. M. Nieminen, J. Phys.: Condens. Matter 5, 3289 (1993).

[41] R. H. Ritchie, W. Brandt, and P. M. Echenique, Phys. Rev. B 14, 4808 (1976).

[42] P. M. Echenique, R. H. Ritchie, and W. Brandt, Phys. Rev. B 20, 2567 (1979). 